\title{
Radial Distribution Function Study for Ethylene-Propylene Random Copolymer by X-Ray Diffraction Method
}

\author{
Mikio NumaKaWA and Akira OdAJIMA \\ Department of Applied Physics, Faculty of Engineering, \\ Hokkaido University, Sapporo 060, Japan.
}

(Received January 16, 1981)

\begin{abstract}
The local chain conformation and packing in the amorphous solid of a polymer were determined for ethylene-propylene random copolymer (EPRC) by the analysis of the radial distribution function (RDF) obtained from X-ray diffraction measurement at room temperature. A new method was devised for removing the contribution of $\mathrm{H}$ atoms to the observed RDF in such a polyatomic system consisting of $\mathrm{C}$ and $\mathrm{H}$ atoms, and the C-C pair RDF for EPRC was derived. From the C-C pair RDF, the number of intrachain and interchain atoms were obtained. From this result, it was found that the amorphous bulk state and the isolated state in $\theta$-solvent are nearly equal in the conformational probabilities for trans and gauche states in the EPRC chain.

KEY WORDS Radial Distribution Function / Ethylene-Propylene

Random Copolymer / Amorphous Solid / C-2H System / C-C Pair RDF /

Conformation / Chain Packing /
\end{abstract}

Radial distribution function (RDF) analysis is a very powerful tool for studying local chain conformation and packing of polymer chains in the amorphous state. The RDF characterizes short range orders in terms of the number of neighboring atoms (the area under the RDF curve) and peak positions. On the other hand, chain conformations are characterized by unconditional probabilities, $P_{t}$ for trans and $P_{g}$ for gauche, and/or higher order probabilities, for instance, $P_{t t}$ and $P_{t g}$, which are defined by a set of rotational angles about the skeletal bonds.

Polyethylene (PE) is the most suitable polymer for such RDF studies because of the simplicity of its molecular structure. But it is difficult to observe the amorphous intensity exactly at room temperature by $\mathrm{X}$-ray diffraction since $\mathrm{PE}$ is a highly crystalline polymer. RDF studies of PE have been made only in the molten or amorphotized states by irradiation, ${ }^{1-6}$ but no quantitative information on the local chain conformation in the amorphous state has yet been obtained from these RDF studies. $\mathrm{RDF}$ of such $\mathrm{C}-2 \mathrm{H}$ systems is governed by $\mathrm{H}$ atoms as well as $\mathrm{C}$ atoms, although the former is not dominant. The intrachain peaks associated with $\mathrm{H}$ atoms are almost smeared out in the RDF pattern obtained from X-ray diffraction measurement. ${ }^{7}$ For such a polyatomic system, it is not easy to obtain exact conformational information from the RDF. It is therefore desirable to derive the number of intrachain atoms only for $\mathrm{C}-\mathrm{C}$ pairs from the $\mathrm{RDF}$ data, by eliminating the contribution from $\mathrm{H}$ atoms.

In this paper, a new method is proposed for obtaining a $\mathrm{C}-\mathrm{C}$ pair RDF from the experimental $\mathrm{RDF}$ of a C-2H system obtained by X-ray diffraction measurement. The local chain conformation and interchain packing for an ethylene-propylene random copolymer (EPRC) with a high ethylene fraction, which is a $\mathrm{C}-2 \mathrm{H}$ system, are studied, because EPRC is in the amorphous solid state at room temperature and its chain structure as simple as $\mathrm{PE}$.

\section{EXPERIMENTAL}

\section{Sample}

A $1 \mathrm{~mm}$ thick film of ethylene-propylene copolymer (propylene-unit fraction; $20 \mathrm{~mol} \%$ ) was provided by the Japan EP Rubber Co. The ${ }^{13} \mathrm{C}$ NMR data show that the populations of propylene sequences $\left(-\mathrm{CH}_{2} \mathrm{CHCH}_{3}-\right)_{n}$ contained are $85 \%$ for $n=1,15 \%$ for $n=2$, and $<1 \%$ for $n>3$. 
From this result, the sample is considered to be a random copolymer, ${ }^{*}$ and is referred to as EPRC. The melting point of the as-received sample is $38^{\circ} \mathrm{C}$ and the glassy transition temperature, $-53^{\circ} \mathrm{C}$. By quenching from the molten state at about $100^{\circ} \mathrm{C}$ to $-20^{\circ} \mathrm{C}$, a completely amorphotized film having a density of $0.865 \mathrm{~g} \mathrm{~cm}^{-3}$ was made.

\section{$X$-Ray Measurement}

X-Ray measurements were carried out at room temperature, using Mo-K $\alpha$ radiation $(\lambda=$ $0.7107 \AA$ ), which was monochromatized by balanced $\mathrm{Zr}$-Yt Ross filters equipped on the incident side. The scintillation counter and pulse height analyzer were employed as a detecting system. The symmetrical transmission technique with a divergence slit of $1 / 6^{\circ}$ was used below $36^{\circ}$ of $2 \theta$ at angular intervals of $0.2^{\circ}$ in $\Delta 2 \theta$, while the symmetrical reflection technique with a divergence slit of $1 / 2^{\circ}$ was used over $28^{\circ}$ of $2 \theta$ at angular intervals of $0.4^{\circ}$ in $\Delta 2 \theta$. Intensity data were collected over an angular range from $2 \theta=2$ to $105^{\circ}$, by point counting in the fixed count mode; the counts per point were $2 \times 10^{4}$ and $8 \times 10^{4}$ for the transmission and reflection techniques, respectively. The intensity curve was made smooth, and then the scattering intensity $I_{\mathrm{obs}}^{\mathrm{c}}(s)$ was corrected for air scattering, absorption, polarization and multiple scattering, ${ }^{8}$ where $s=4 \pi \sin \theta / \lambda$.

\section{EXPERIMENTAL RESULTS}

\section{Intensity Curve}

Figure 1 shows the observed coherent intensity curve $I(s)$ in electron units obtained by removing the incoherent scattering. In order to transform the intensity in an arbitrary unit to that in electron units, the normalization factor was determined by the following high angle method $^{9}$ :

$$
\begin{aligned}
\beta= & \int_{s_{\mathrm{high}}}^{s_{\text {max }}}\left[I_{\mathrm{C}}^{\mathrm{inc}}(s)+2 I_{\mathrm{H}}^{\mathrm{inc}}(s)+f_{\mathrm{C}}^{2}(s)+2 f_{\mathrm{H}}^{2}(s)\right] \mathrm{d} s / \\
& \int_{s_{\text {high }}}^{s_{\max }} I_{\mathrm{obs}}^{\mathrm{c}}(s) \mathrm{d} s
\end{aligned}
$$

* A Monte Carlo chain of 500 monomers consisting of ethylene and propylene units $(20 \mathrm{~mol} \%$ of propylene unit) indicated that the populations of propylene sequences were $84 \%$ for $n=1,16 \%$ for $n=2$, and $0 \%$ for $n>3$. where $s_{\text {high }}=10 \AA^{-1}$ and $s_{\max }=14 \AA^{-1}, I_{\mathrm{i}}^{\text {inc }}(s)$ and $f_{\mathrm{i}}(s)$ are the incoherent scattering intensity and the scattering factor for the $\mathrm{i}$-type atom $(\mathrm{i}=\mathrm{C}$ (carbon) or $\mathrm{H}$ (hydrogen)). ${ }^{10}$ The normalization factor calculated by the RDF method ${ }^{9}$ showed only a deviation of $0.02 \%$ from the value calculated by eq 1 . Since EPRC is a random copolymer consisting of ethylene units $\left(-\mathrm{CH}_{2}-\mathrm{CH}_{2}-\right)$ and propylene units $\left(-\mathrm{CH}_{2}-\right.$ $\mathrm{CHCH}_{3}-$ ), it can be treated as a $\mathrm{C}-2 \mathrm{H}$ system in $\mathrm{X}$ ray scattering. For the $\mathrm{C}-2 \mathrm{H}$ system, the $s$-weighted normalized intensity $s i(s)$ is given by,

$$
s i(s)=\left[I(s)-f_{\mathrm{C}}{ }^{2}(s)-2 f_{\mathrm{H}}{ }^{2}(s)\right] /\left[f_{\mathrm{C}}(s)+2 f_{\mathrm{H}}(s)\right]^{2}
$$

The sil(s) curve is shown in Figure 2.

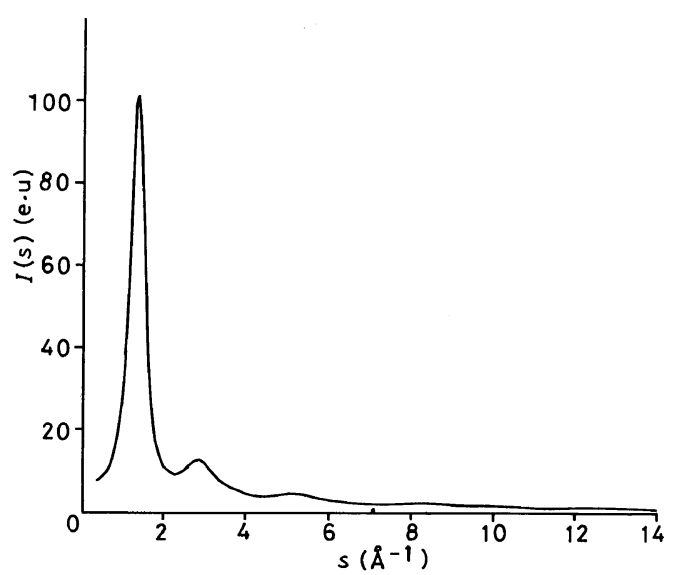

Figure 1. Coherent scattering intensity in an electron unit $I(s)$ for ethylene-propylene random copolymer, EPRC (propylene mole fraction $=20 \%$ ) measured at room temperature.

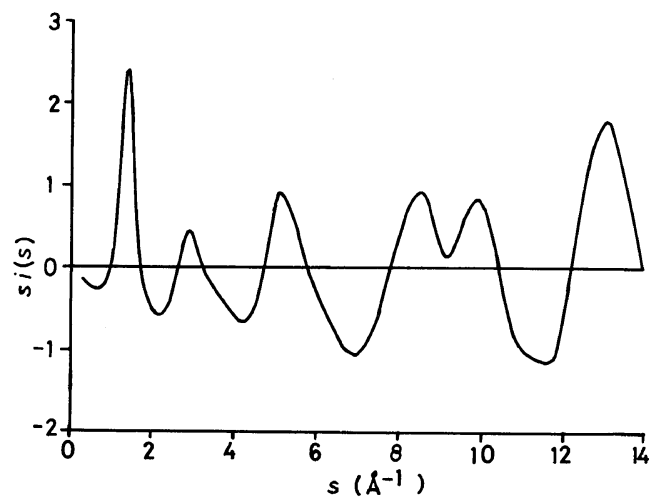

Figure 2. $s$-weighted normalized intensity $s i(s)$ for EPRC, obtained from the coherent scattering intensity at room temperature. 
RDF Study for Ethylene Propylene Random Copolymer
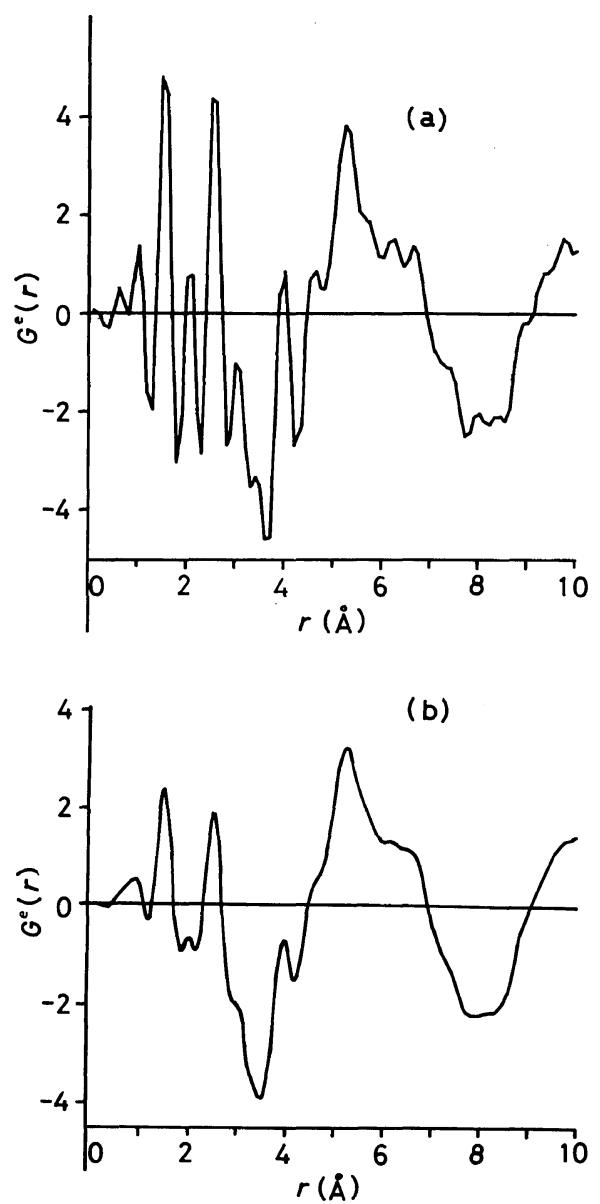

Figure 3. Experimental DRDF $G^{\mathrm{e}}(r)$ for EPRC at room temperature, obtained from $s i(s)$; (a) without a damping factor and (b) with a damping factor of $\exp \left(-0.01 s^{2}\right)$.

\section{$D R D F$ and $R D F$}

The experimental differential radial distribution function (DRDF) $G^{\mathrm{e}}(r)$ is obtained from the $s i(s)$ data in Figure 2 using the following equation:

$$
G^{\mathrm{e}}\left(r_{j}\right)=2 r_{j} / \pi \sum_{s_{k}=s_{\min }}^{s_{\max }} s_{k} i\left(s_{k}\right) \cdot \exp \left(-\gamma s_{k}^{2}\right) \sin \left(r_{j} s_{k}\right) \Delta s
$$

where

$$
s_{\min }=0.35 \AA^{-1}, \Delta s=0.05 \AA^{-1} \text {, and } \exp \left(-\gamma s_{k}^{2}\right)
$$

is a damping factor to minimize truncation errors. Intervals of $r_{j}$ in DRDF are $0.05 \mathrm{~A}$ for $r<6 \AA$ and $0.1 \mathrm{~A}$ for $r>6 \AA$. Figure $3 \mathrm{a}$ shows the $G^{\mathrm{e}}(r)$ obtained from the $s i(s)$ curve in Figure 2 with no damping factor, i.e., with $\gamma=0.0$. There exist
Table I. Intrachain distances of $C_{0} C_{j}$ pairs separated by $j$ bonds ( $j=1$ to 4 ) for a linear polyethylene chain

\begin{tabular}{cccc}
\hline \multirow{2}{*}{ Bond $j$} & $\begin{array}{c}C_{0} C_{j} \text { distance } \\
\end{array}$ & $\begin{array}{c}\text { Confor- } \\
\text { mation }\end{array}$ & $C_{0} H_{j}$ distance \\
\hline 1 & 1.54 & & $\AA$ \\
2 & 2.54 & & 1.09 \\
3 & 3.01 & $g^{ \pm}$ & $\left\{\begin{array}{l}2.77 \\
3.48\end{array}\right.$ \\
& 3.91 & $t$ & 2.77 \\
& 3.63 & $g^{ \pm} g^{ \pm}$ & $\left\{\begin{array}{l}2.63 \\
4.00\end{array}\right.$ \\
& 4.43 & $t g^{ \pm}$ & $\left\{\begin{array}{l}4.20 \\
4.69\end{array}\right.$ \\
& 5.08 & $t t$ & 4.19 \\
\hline
\end{tabular}

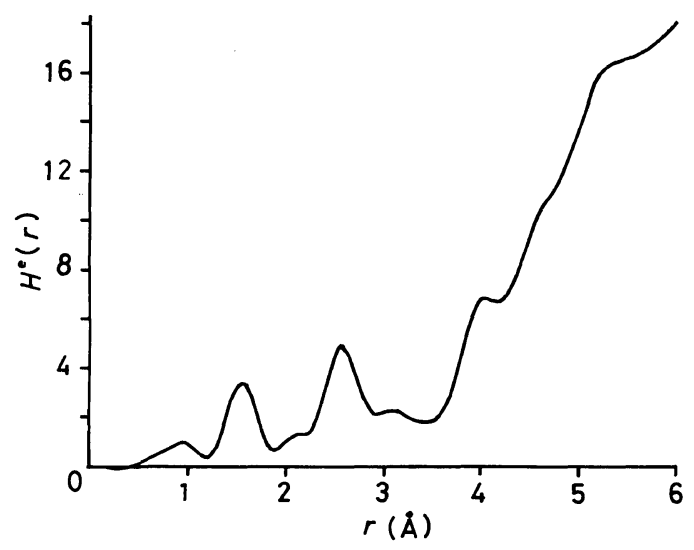

Figure 4. Experimental RDF $H^{\mathrm{e}}(r) \quad\left(=G^{\mathrm{e}}(r)+4 \pi r^{2} \bar{\rho}\right)$ for EPRC at room temperature.

many peaks in $G^{\mathrm{e}}(r)$, but most of them are considered to be suprious peaks due to truncation errors. At first sight, many peaks within $6 \AA$ seem to correspond to the intrachain- $\mathrm{CC}$ and $-\mathrm{CH}$ distances which were assigned by Voigt-Martin et al. ${ }^{1}$ in the DRDF of molten PE obtained from electron diffraction measurement. Longman et al. ${ }^{2}$ made a similar finding from X-ray diffraction measurement, and suggested that such peaks are due to intrachain the $\mathrm{CC}$ and $\mathrm{CH}$ distances themselves and truncation errors are not serious in the region of $1<r<6 \AA$. In our previous paper, ${ }^{7}$ however, it was indicated that the intrachain $\mathrm{CH}$ peaks should be smeared out 
when DRDF was obtained from the X-ray scattering experiment, since the scattering intensity from $\mathrm{H}$ atoms decreases rapidly with an increase in $s$. It was also indicated that the use of a damping factor $\exp \left(-0.01 s^{2}\right)$ almost extinguishes spurious peaks, which are caused by the finite truncation of $s_{\max }=14 \AA^{-1}$, without overdamping true peaks. Figure $3 \mathrm{~b}$ shows the $G^{\mathrm{e}}(r)$ obtained by applying the damping factor of $\exp \left(-0.01 s^{2}\right)$. Intrachain CC and $\mathrm{CH}$ distances calculated for a linear $\mathrm{PE}$ chain are shown in Table I. In Figure 3b, intrachain $\mathrm{CH}$ peaks have almost disappeared, while intrachain CC peaks appear at $1.55 \AA, 2.55 \AA$, and $4.0 \AA$. A shoulder appearing at about $3 \AA$ corresponds to the gauche $\mathrm{CC}$ distance given in Table I. Figure 4 shows $\operatorname{RDF} H^{\mathrm{e}}(r)\left(G^{\mathrm{e}}(r)+4 \pi r^{2} \bar{\rho}\right)$, where $\bar{\rho}$ is the average number of $\mathrm{C}$ atoms per unit volume. The value of $\bar{\rho}$, calculated from the macroscopic sample density, was $0.0371 \AA^{-3}$.

\section{DATA ANALYSIS}

\section{Scattering Intensity from a $\mathrm{C}-2 \mathrm{H}$ System}

The coherent scattering from a $\mathrm{C}-2 \mathrm{H}$ system is given by

$$
\begin{aligned}
I(s)= & f_{\mathrm{C}}^{2}(s)+2 f_{\mathrm{H}}^{2}(s)+f_{\mathrm{C}}{ }^{2}(s) \int_{0}^{\infty} g_{\mathrm{CC}}^{0}(r) \sin (s r) /(s r) \mathrm{d} r \\
& +4 f_{\mathrm{C}}(s) f_{\mathrm{H}}(s) \int_{0}^{\infty} g_{\mathrm{CH}}^{0}(r) \sin (s r) /(s r) \mathrm{d} r \\
& +4 f_{\mathrm{H}}{ }^{2}(s) \int_{0}^{\infty} g_{\mathrm{HH}}^{0}(r) \sin (s r) /(s r) \mathrm{d} r
\end{aligned}
$$

where the $\mathrm{i}-\mathrm{j}$ pair DRDF $g_{\mathrm{ij}}^{0}(r)$ is defined by $4 \pi r^{2}\left(\rho_{\mathrm{ij}}^{0}(r)-\bar{\rho}\right)$, with $\rho_{\mathrm{ij}}^{0}(r)$ the number density of $\mathrm{j}$ type atoms at a distance $r$ from an i type atom.

DRDF $G(r)$ is obtained by the inverse Fourier transform of $s i(s)$,

$$
G(r)=2 r / \pi \int_{s_{\min }}^{s_{\max }} s i(s) \sin (r s) \cdot \exp \left(-\gamma s^{2}\right) \mathrm{d} s
$$

where $s i(s)$ is defined by eq 2 . If $f_{\mathrm{H}}(s) / f_{\mathrm{C}}(s)$ is a constant, the scattering factors can be expressed as $f_{\mathrm{C}}(s)=Z_{\mathrm{C}} f_{\mathrm{e}}(s)$ and $f_{\mathrm{H}}(s)=Z_{\mathrm{H}} f_{\mathrm{e}}(s)$, where $f_{\mathrm{e}}(s)=$ $\left[f_{\mathrm{C}}(s)+2 f_{\mathrm{H}}(s)\right] /\left(Z_{\mathrm{C}}+2 Z_{\mathrm{H}}\right)$ and $Z_{\mathbf{i}}$ is the atomic number of the i type atom. In this case, $G(r)$ reduces to the weighted sum of the $i-j$ pair DRDF $g_{\mathrm{ij}}(r)^{*}$, i.e., $G^{\mathrm{t}}(r)$. Thus,

$$
\begin{aligned}
G(r)=G^{\mathrm{t}}(r)= & {\left[Z_{\mathrm{C}}^{2} g_{\mathrm{CC}}(r)+4 Z_{\mathrm{C}} Z_{\mathrm{H}} g_{\mathrm{CH}}(r)\right.} \\
& \left.+4 Z_{\mathrm{H}}^{2} g_{\mathrm{HH}}(r)\right] /\left(Z_{\mathrm{C}}+2 Z_{\mathrm{H}}\right)^{2}
\end{aligned}
$$

When $f_{\mathrm{H}}(s) / f_{\mathrm{C}}(s)$ is not a constant, however, it is not easy to derive meaningful information on the pair distribution from $G(r)$. We treat the X-ray scattering in this case as follows. The scattering factors are expressed as $f_{\mathrm{C}}(s)=Z_{\mathrm{C}} f_{\mathrm{e}}(s)+\Delta f_{\mathrm{C}}(s)$ and $f_{\mathrm{H}}(s)=Z_{\mathrm{H}} f_{\mathrm{e}}(s)+\Delta f_{\mathrm{H}}(s)$.

Substituting these $f_{\mathrm{C}}(s)$ and $f_{\mathrm{H}}(s)$ into eq 4 , we obtain the following relations:

$s i(s)=s i_{0}(s)+\Delta s i(s)$

$$
\begin{aligned}
s i_{0}(s)= & \int_{0}^{\infty}\left\{\left[Z_{\mathrm{C}}^{2} g_{\mathrm{CC}}^{0}(r)+4 Z_{\mathrm{C}} Z_{\mathrm{H}} g_{\mathrm{CH}}^{0}(r)+4 Z_{\mathrm{H}}^{2} g_{\mathrm{HH}}^{0}(r)\right]\right. \\
& \left./\left[r\left(Z_{\mathrm{C}}+2 Z_{\mathrm{H}}\right)^{2}\right]\right\} \sin (s r) \mathrm{d} r
\end{aligned}
$$

and,

$$
\begin{aligned}
\Delta s i(s)= & F_{\mathrm{CC}}(s) \int_{0}^{\infty}\left[g_{\mathrm{CC}}^{0}(r) / r\right] \sin (s r) \mathrm{d} r \\
& +4 F_{\mathrm{CH}}(s) \int_{0}^{\infty}\left[g_{\mathrm{CH}}^{0}(r) / r\right] \sin (s r) \mathrm{d} r \\
& +4 F_{\mathrm{HH}}(s) \int_{0}^{\infty}\left[g_{\mathrm{HH}}^{0}(r) / r\right] \sin (s r) \mathrm{d} r
\end{aligned}
$$

where,

$$
\begin{aligned}
F_{\mathrm{ij}}(s)= & \left\{f_{\mathrm{e}}(s)\left[Z_{\mathrm{i}} \Delta f_{\mathrm{j}}(s)+Z_{\mathrm{j}} \Delta f_{\mathrm{i}}(s)\right]+\Delta f_{\mathrm{i}}(s) \Delta f_{\mathrm{j}}(s)\right\} / \\
& \left\{f_{\mathrm{C}}(s)+2 f_{\mathrm{H}}(s)\right]^{2}
\end{aligned}
$$

$F_{\mathrm{CC}}(s)$ and $F_{\mathrm{CH}}(s)$ were calculated numerically from the theoretical values of $f_{\mathrm{C}}(s)$ and $f_{\mathrm{H}}(s)^{10}$; the calculated results are shown by the solid lines in Figure 5. We can approximate $F_{\mathrm{CC}}(s)$ and $F_{\mathrm{CH}}(s)$ by the simple function,

$$
F_{\mathrm{ij}}(s)=K_{\mathrm{ij}}\left[1-\exp \left(-a_{\mathrm{ij}} s^{2}\right)\right]
$$

where $K_{\mathrm{CC}}=0.425, a_{\mathrm{CC}}=0.025 \AA^{-2}, K_{\mathrm{CH}}=-0.091$, and $a_{\mathrm{CH}}=0.024 \AA^{-2}$. The open circles in Figure 5 show such approximations for $F_{\mathrm{CC}}(s)$ and $F_{\mathrm{CH}}(s)$. Substituting eq 7-8 into eq 5 , we derive RDF $H(r)$ $\left(=G(r)+4 \pi r^{2} \bar{\rho}\right)$ :

* $g_{\mathrm{ij}}(r)$ in eq 6 is different from $g_{\mathrm{ij}}^{0}(r)$. The former is smeared out by the truncation effect, and hereafter is referred to as $\mathrm{i}-\mathrm{j}$ pair DRDF. 
RDF Study for Ethylene Propylene Random Copolymer
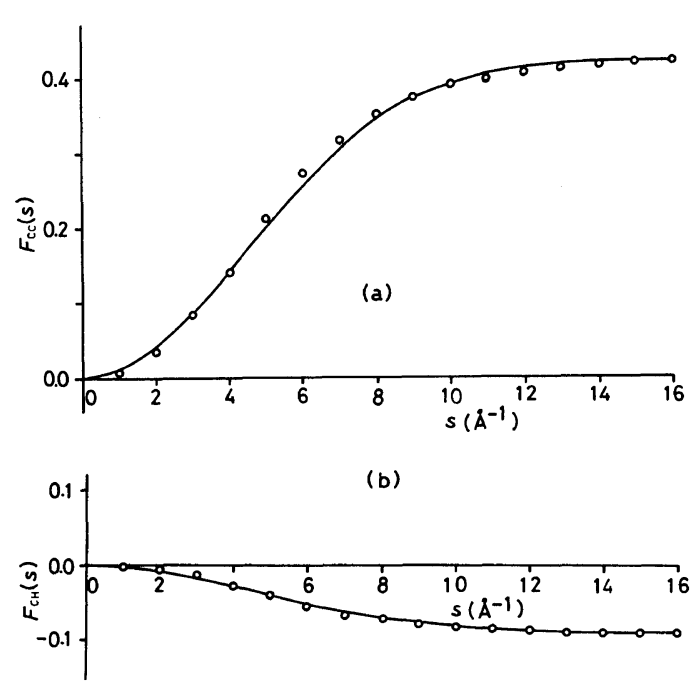

Figure 5. Correction terms for scattering factors, (a) $F_{\mathrm{CC}}(s)$ and (b) $F_{\mathrm{CH}}(s)$. Solid line are calculated numerically by using the theoretical values of $f_{\mathrm{C}}(s)$ and $f_{\mathrm{H}}(s)$. Open circles show approximations of $F_{\mathrm{ij}}(s)$ 's expressed by simple functions in eq 9 .

$$
H(r)=H_{\mathrm{CC}}(r)+H_{\mathrm{CH}}(r)
$$

Here,

$$
\begin{aligned}
H_{\mathrm{CC}}(r)= & {\left[Z_{\mathrm{C}}^{2} /\left(Z_{\mathrm{C}}+2 Z_{\mathrm{H}}\right)^{2}\right] h_{\mathrm{CC}}(r)+\Delta H_{\mathrm{CC}}(r) } \\
H_{\mathrm{CH}}(r)= & 4\left[Z_{\mathrm{C}} Z_{\mathbf{H}} /\left(Z_{\mathrm{C}}+2 Z_{\mathrm{H}}\right)^{2}\right] h_{\mathrm{CH}}(r) \\
& +4 \Delta H_{\mathrm{CH}}(r)
\end{aligned}
$$

and $H_{\mathrm{HH}}(r)$ is neglected, where the $\mathrm{i}-\mathrm{j}$ pair RDF $h_{\mathrm{ij}}(r)$ is defined by $4 \pi r^{2} \rho_{\mathrm{ij}}(r) . H_{\mathrm{ij}}(r)$ is hereafter referred to as atomic-number-weighted $\mathrm{i}-\mathrm{j}$ pair RDF. The second terms in eq $11 \mathrm{a}$ and $\mathrm{b}$ are given by,

$$
\begin{aligned}
\Delta H_{\mathrm{ij}}(r) & =r f_{\mathrm{ij}}(r)^{*}\left[h_{\mathrm{ij}}(r) / r\right] \\
f_{\mathrm{ij}}(r) & =1 / \pi \int_{0}^{\infty} F_{\mathrm{ij}}(s) \cos (r s) \mathrm{d} s
\end{aligned}
$$

where ${ }^{*}$ denotes the convolution product.

\section{C-C Pair RDF}

In order to make clear local conformation as well as local packing in the amorphous EPRC, the smeared-out $\mathrm{CH}$ peaks were removed from the experimental RDF $H^{\mathrm{e}}(r)$ by the following procedures:

i) The conformational probabilities for the
Table II. Conformational probabilities calculated for an isolated EPRC chain (proplyene mole fraction $=20 \%$ ) at room temperature

\begin{tabular}{ll}
\hline$P_{t}$ & 0.59 \\
$P_{g^{ \pm}}$ & 0.41 \\
\hline$P_{t t}$ & 0.36 \\
$P_{t g^{ \pm}}$ & 0.54 \\
$P_{g^{ \pm} g^{ \pm}}$ & 0.10 \\
\hline
\end{tabular}

EPRC chain (propylene mole fraction $=20 \%$ ) were calculated on the rotational isomeric state model for trans $(t)$, gauche $\left(g^{ \pm}\right), t t, t g^{ \pm}, g^{ \pm} g^{ \pm}$, as shown in Table II. (The outline of calculation is described in Appendix 1.) The intrachain $\mathrm{C}-\mathrm{H}$ pair $\mathrm{RDF}$ $h_{\mathrm{CH}}^{0 \text { intra }}(r)$ was obtained from the conformational probabilities and the $\mathrm{C}_{0} \mathrm{H}_{j}$ distances separated by $j$ bonds in the chain. If the intrachain $\mathrm{C}-\mathrm{H}$ pair RDF is obtained from the observed $s i(s)$ by eq 5 , its distribution must be broadened by a finite $s_{\max }$ and the damping factor. According to Warren, ${ }^{11}$ such an intrachain $\mathrm{C}-\mathrm{H}$ pair $\mathrm{RDF} h_{\mathrm{CH}}^{\text {intra }}(r)$ can be calculated from $h_{\mathrm{CH}}^{0 \text { intra }}(r)$ by the following equation:

$$
\begin{aligned}
h_{\mathrm{CH}}^{\mathrm{intra}}(r) & =r s_{\max } / \pi \int_{0}^{\infty} h_{\mathrm{CH}}^{0 \text { intra }}(y) / y\left[\frac{0.588 \sin (r-y) s_{\max }}{(r-y) s_{\max }}\right. \\
& +0.206 \frac{\sin (2.15 \gamma+|r-y|) s_{\max }}{(2.15 \gamma+|r-y|) s_{\max }} \\
& \left.+0.206 \frac{\sin (2.15 \gamma-|r-y|) s_{\max }}{(2.15 \gamma-|r-y|) s_{\max }}\right] \mathrm{d} y
\end{aligned}
$$

where we have used $s_{\max }=14 \AA^{-1}$ and $\gamma=0.01$ as described in the experimental part (cf. eq 3). For the $\mathrm{C}_{0} \mathrm{H}_{j}$ pair distances $(j=1$ to 4$)$, the values for a linear PE chain were used as shown in Table I. Since the $\mathrm{CH}$ peaks are smeared out in $H_{\mathrm{CH}}^{\mathrm{intra}}(r)$ because $f_{\mathrm{H}}(s) / f_{\mathrm{C}}(s)$ is not constant, the use of these distances for the EPRC chain yields no serious error. $H_{\mathrm{CH}}^{\mathrm{intra}}(r)$, calculated from $h_{\mathrm{CH}}^{\mathrm{intra}}(r)$, is shown in Figure $6 \mathrm{a}$. Figure $6 \mathrm{~b}$ indicates the result when $H_{\mathrm{CH}}^{\text {intra }}(r)$ is subtracted from $H^{\mathrm{e}}(r)$, or $H^{\mathrm{e}}(r)-H_{\mathrm{CH}}^{\mathrm{intra}}(r)$. This is equal to $H_{\mathrm{CC}}(r)$ (the atomic-number-weighted $\mathrm{C}-\mathrm{C}$ pair $\mathrm{RDF}$ ) in a small $r$ region. However, a tail of the interchain RDF $H^{\text {inter }}(r)$ still extends to the region below $4 \AA$.

ii) In view of van der Waals contacts, the tail part of $H^{\text {inter }}(r)$ is given by the dotted line shown in Figure $6 \mathrm{~b}$. The intrachain $\mathrm{CC}$ peaks have been 
separated from $H^{\mathrm{e}}(r)-H_{\mathrm{CH}}^{\mathrm{intra}}(r)$ by drawing the dotted line, in such a way that the resultant area of intrachain peaks in the range of 2.2 to $4.3 \AA$ is equal to the theoretical value predicted for the EPRC chain, $4.4 \times Z_{\mathrm{C}}^{2} /\left(Z_{\mathrm{C}}+2 Z_{\mathrm{H}}\right)^{2 *}$. As a result, the dotted line intersects the $H^{\mathrm{e}}(r)-H_{\mathrm{CH}}^{\mathrm{intra}}(r)$ curve at about $3.4 \AA$ and $3.7 \AA$ of $r$. The dashed line in Figure $6 \mathrm{~b}$ shows another separation for avoiding such an intersection, and the area of the intrachain peaks is also taken to be the theoretical value.

In a large $r$ region, $\mathrm{CH}_{x}$ units $(x=1$ to 3$)$ are assumed to be distributed with random orientation since most of $\mathrm{CH}_{x}$ units belong to different chain segments. Moreover, a C-H distance $\left(r_{\mathrm{CH}}=1.09 \AA\right)$ in the $\mathrm{CH}_{x}$ unit is much smaller than interchain radial distances of $\mathrm{H}$ and/or $\mathrm{C}$ atoms. Therefore, it is assumed that $\mathrm{C}$ and $\mathrm{H}$ atoms belonging to the same $\mathrm{CH}_{x}$ unit are distributed radially in the same form in the large $r$ region. Therefore, interchain $\mathrm{i}-\mathrm{j}$ pair DRDF $g_{\mathrm{ij}}^{0 \mathrm{inter}}(r)$ is given by the following relations:

$$
\begin{array}{ll}
g_{\mathrm{CC}}^{0 \text { inter }}(r)=g_{\mathrm{CH}}^{0 \text { inter }}(r)=g_{\mathrm{HH}}^{0 \text { inter }}(r) & \text { for } \quad r>r_{0} \\
g_{\mathrm{ij}}^{0 \text { inter }}(r)=0 & \text { for } \quad r<r_{0}
\end{array}
$$

and

$$
g_{\mathrm{ij}}^{0}(r)=g_{\mathrm{ij}}^{0 \text { inter }}(r)+g_{\mathrm{ij}}^{0 \text { intra }}(r)
$$

where $r_{0}$ is the nearest distance of interchain atoms. When such relations are taken into consideration in eq 4 , the coherent intensity is given by,

$$
\begin{aligned}
I(s)= & f_{\mathrm{C}}^{2}(s)+2 f_{\mathrm{H}}^{2}(s) \\
& +\left[f_{\mathrm{C}}(s)+2 f_{\mathrm{H}}(s)\right]^{2} \int_{0}^{\infty} g_{\mathrm{CC}}^{0}{ }^{\text {inter }}(r) \sin (s r) /(s r) \mathrm{d} r \\
& +\sum_{\mathrm{ij}} \chi_{\mathrm{i}} \chi_{\mathrm{j}} f_{\mathrm{i}}(s) f_{\mathrm{j}}(s) \int_{0}^{\infty} g_{\mathrm{ij}}^{0 \text { intra }}(r) \sin (s r) /(s r) \mathrm{d} r(14)
\end{aligned}
$$

where $\chi_{\mathrm{i}}$ is the atomic fraction of the i-type atom. The inverse Fourier transform of $s i(s)$, obtained from eq 5 , then gives $G^{\text {inter }}(r)=g_{\text {CC }}^{\text {inter }}(r)$ for $r>r_{0}$, where $G^{\text {inter }}(r)$ denotes the interchain part of $G(r)$. $G(r) \simeq G^{\text {inter }}(r)$ can be assumed in the large $r$ region. Therefore, $H^{\mathrm{e}}(r)-H_{\mathrm{CH}}^{\mathrm{intra}}(r)$ above $4.3 \AA$ as well as the dashed or dotted lines below $4.3 \AA$ in Figure $6 \mathrm{~b}$ can be considered to be the interchain $\mathrm{C}-\mathrm{C}$ pair

* The area of $\mathrm{C}_{0} \mathrm{C}_{2}$ and/or $\mathrm{C}_{0} \mathrm{C}_{3}$ pair in the atomicnumber-weighted $\mathrm{C}-\mathrm{C}$ pair $\mathrm{RDF} H_{\mathrm{CC}}(r)$ is given by $2.2 \times Z_{\mathrm{C}}^{2} /\left(Z_{\mathrm{C}}+2 Z_{\mathrm{H}}\right)^{2}$.
$\mathrm{RDF} h_{\mathrm{CC}}^{\mathrm{inter}}(r)$ in the first approximation.

iii) For the second approximation, the atomicnumber-weighted $\mathrm{C}-\mathrm{H}$ pair $\mathrm{RDF}$ for interchain $H_{\mathrm{CH}}^{\text {inter }}(r)$ must be derived more exactly. If $\mathrm{H}$ atoms are distributed with a spherical symmetry about the $\mathrm{C}$ atom in the same $\mathrm{CH}_{x}$ unit, interchain $\mathrm{C}-\mathrm{H}$ pair RDF can be obtained from $h_{\mathrm{CC}}^{\text {inter }}(r)$, i.e.,

$$
h_{\mathrm{CH}}^{\text {inter }}(r)=\int_{0}^{\infty} h_{\mathrm{CH}}\left(r, r_{\mathrm{C}}\right) h_{\mathrm{CC}}^{\text {inter }}\left(r_{\mathrm{C}}\right) \mathrm{d} r_{\mathrm{C}}
$$

where $h_{\mathrm{CH}}\left(r, r_{\mathrm{C}}\right)$ is the RDF of an $\mathrm{H}$ atom when the $\mathrm{C}$ atom in the same $\mathrm{CH}_{x}$ unit is located at $r_{\mathrm{C}}$. The derivation of $h_{\mathrm{CH}}\left(r, r_{\mathrm{C}}\right)$ is described in Appendix 2. $H_{\mathrm{CH}}^{\text {inter }}(r)$ obtained from $h_{\mathrm{CH}}^{\text {inter }}(r)$ using eq $11 \mathrm{~b}-\mathrm{d}$ is shown in Figure 6c. Two cases were obtained for $H_{\mathrm{CH}}^{\mathrm{inter}}(r)$ : One is from the dotted line and the other from the dashed line, for $h_{\mathrm{CH}}^{\text {inter }}(r)$ below $4.3 \AA$ in Figure 6b. However, both $H_{\mathrm{CH}}^{\mathrm{inter}}(r)$ 's were undistinguishably coincident with each other. This may be due to the fact that $\mathrm{H}$ atoms are distributed spherically symmetrically about $\mathrm{C}$ atom as given by eq 14 and $H_{\mathrm{CH}}^{\mathrm{inter}}(r)$ is furthermore smeared out because $f_{\mathrm{H}}(s) / f_{\mathrm{C}}(s)$ is not constant. Therefore, inasmuch as the area of $h_{\mathrm{CC}}^{\text {inter }}(r)$ stays the same, almost the same $H_{\mathrm{CH}}^{\mathrm{inter}}(r)$ is derived from $h_{\mathrm{CC}}^{\text {inter }}(r)$. The experimental atomic-number-weighted C-C pair RDF $H_{\mathrm{CC}}^{\mathrm{e}}(r)$ was obtained by subtracting the $H_{\mathrm{CH}}^{\text {inter }}(r)$ from $H^{\mathrm{e}}(r)-H_{\mathrm{CH}}^{\mathrm{intra}}(r)$; the result is shown in Figure 6d.

iv) The experimental C-C pair $\mathrm{RDF} h_{\mathrm{CC}}^{\mathrm{e}}(r)$ was obtained using the iterative method as follows. From eq $11 \mathrm{~b}$, the relation between $\Delta H_{\mathrm{CC}}(r)$ and $h_{\mathrm{CC}}^{\mathrm{e}}(r)$ for the $n$-th interation is given by,

$$
h_{\mathrm{CC}}^{\mathrm{e}(n)}(r)=\left[\left(Z_{\mathrm{C}}+2 Z_{\mathrm{H}}\right)^{2} / Z_{\mathrm{C}}{ }^{2}\right]\left[H_{\mathrm{CC}}^{\mathrm{e}}(r)-\Delta H_{\mathrm{CC}}^{(n)}(r)\right]
$$

If $h_{\mathrm{CC}}^{\mathrm{e}(n-1)}(r)$ is known, $\Delta H_{\mathrm{CC}}^{(n)}(r)$ is calculated from the $h_{\mathrm{CC}}^{\mathrm{e}(n-1)}(r)$ using eq $11 \mathrm{c}$. $\left[\left(Z_{\mathrm{C}}+2 Z_{\mathrm{H}}\right)^{2} / Z_{\mathrm{C}}{ }^{2}\right] H_{\mathrm{CC}}^{\mathrm{e}}(r)$ was used as $h_{\mathrm{CC}}^{\mathrm{e}(0)}(r)$, and $h_{\mathrm{CC}}^{\mathrm{e}(n)}(r)$ converged to a certain function after iterations were carried out several times. For $n=11, \Delta H_{\mathrm{CC}}^{\mathrm{e}(n)}(r)$ and $h_{\mathrm{CC}}^{\mathrm{e}(n)}(r)$ are shown in Figure 6e and $f$, respectively.

The dotted lines in Figure $6 \mathrm{f}$ show the peak separations at about $1.5 \AA, 2.5 \AA$, and $3 \AA$ plus $4 \AA$. These separations were carried out in such a way that the number of atoms in $1.5 \AA, 2.5 \AA$, and $3 \AA$ plus $4 \AA$ peaks were $2.0,2.2$, and 2.2 , respectively, as predicted from the EPRC chain structure (propylene-unit fraction: $20 \mathrm{~mol}^{\%} \%$ ) presented in the experimental part. Furthermore, each of the $3 \AA$ and $4 \AA$ peaks was separated, as shown by the 

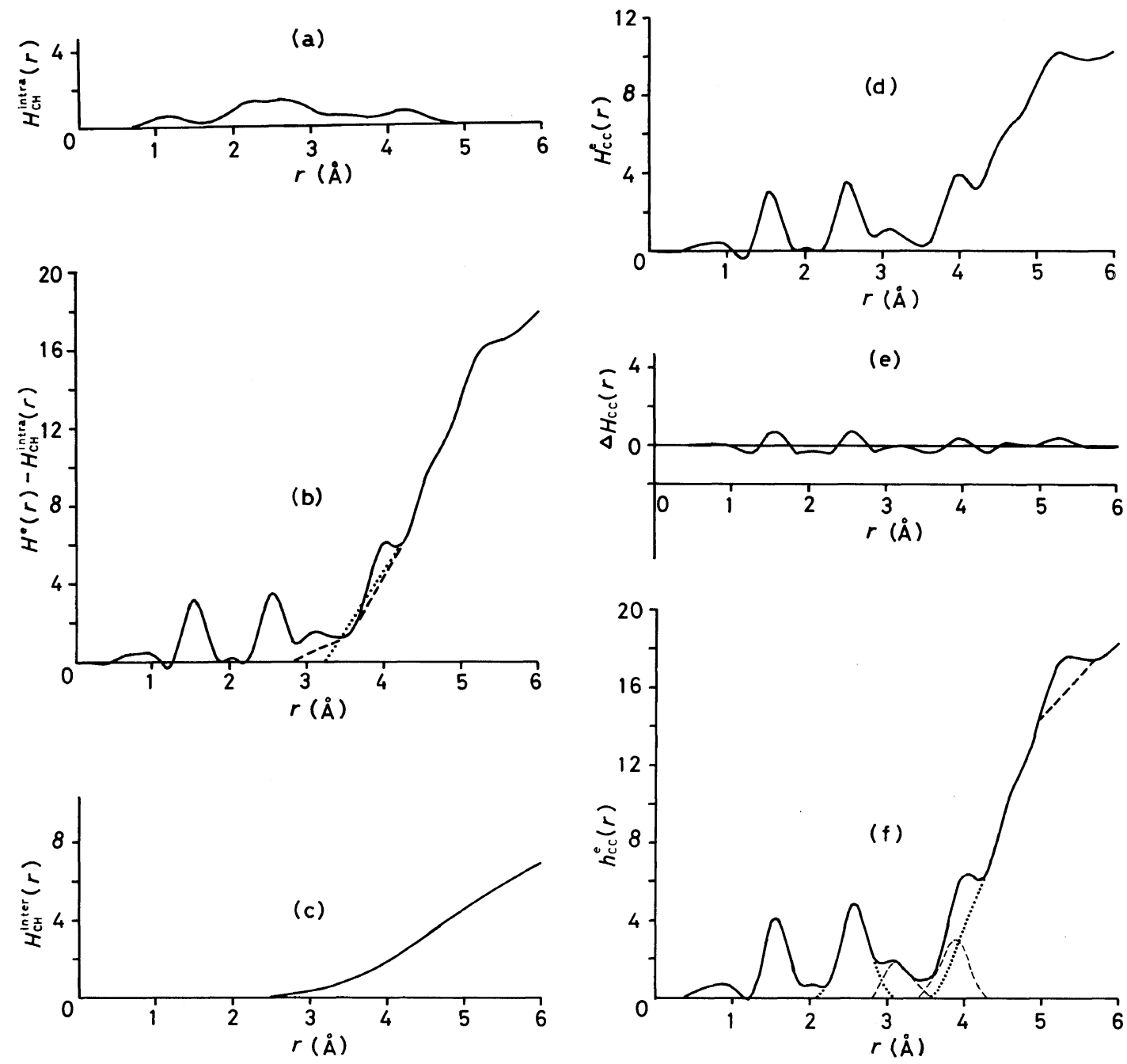

Figure 6. (a) Atomic-number-weighted $\mathrm{C}-\mathrm{H}$ pair RDF for intrachain, $H_{\mathrm{CH}}^{\mathrm{intra}}(r)$, as a function of $r$. (b) Removal of intrachain $\mathrm{H}$ atoms from $H^{\mathrm{e}}(r) ; H^{\mathrm{e}}(r)-H_{\mathrm{CH}}^{\mathrm{intra}}(r)$. (c) Atomic-number-weighted C-H pair RDF for interchain, $H_{\mathrm{CH}}^{\mathrm{inter}}(r)$. (d) Experimental atomic-number-weighted $\mathrm{C}-\mathrm{C}$ pair $\mathrm{RDF}, H_{\mathrm{CC}}^{\mathrm{e}}(r)$, that is, $H^{\mathrm{e}}(r)-H_{\mathrm{CH}}^{\mathrm{inta}}(r)-H_{\mathrm{CH}}^{\mathrm{inter}}(r)$. (e) Additional term in $H_{\mathrm{CC}}^{\mathrm{e}}(r) ; \Delta H_{\mathrm{CC}}(r)$, caused because $f_{\mathrm{H}}(s) / f_{\mathrm{C}}(s)$ is not constant. (f) Experimental C-C pair RDF, $H_{\mathrm{CC}}(r)$, at room temperature.

dashed line in Figure 6f. The number of atoms in the $3 \AA$ peak is 0.85 , while that of $4 \AA$ peak is 1.35 . The ratio of the number of atoms in the $3 \AA$ peak to that in $4 \AA$ peak (trans-gauche ratio) is about $0.4 / 0.6$. On the other hand, the trans-gauche ratio of an isolated EPRC chain in a $\theta$-solvent was calculated to be $0.41 / 0.59$ at room temperature, as shown in Table II. We see good agreement between the observed and calculated values. It should be noted that the trans-gauche ratio in the amorphous bulk is nearly equal to that in the isolated chain.

Peaks above $4 \AA$ are observed at about 4.5 and $5.3 \AA$ in the $\mathrm{C}-\mathrm{C}$ pair RDF. The peaks at 4.5 and $5.3 \AA$ correspond to the $t g^{ \pm}$and $t t$ states of the $\mathrm{C}_{0} \mathrm{C}_{4}$ pair, respectively. The dashed line about $5.3 \AA$ shows an example of the separation of an intrachain peak from the interchain background, in which separation was performed when the number of atoms in the $5.3 \AA$ peak was equal to the value $(0.8=2.2 \times 0.36)$ calculated from the EPRC chain 
structure and the probability of $t t$ conformation $\left(P_{t t}\right)$ in Table II. This suggests that the sharp peak appearing at $5.2 \AA$ in the observed $G^{\mathrm{e}}(r)$, shown in Figure $3 \mathrm{~b}$, is contributed primarily from the intrachain-t $t$-conformation.

As shown by the dotted line in Figure 6f, the closest possible distance of atoms in the neighboring chains is about $3.6 \AA$, although some arbitrariness in separation was unavoidable. This value may increase a little if the truncation broadening in the tail part of interchain region of $h_{\mathrm{CC}}^{\mathrm{e}}(r)$ is corrected. The number of interchain atoms is also estimated from $h_{\mathrm{CC}}^{\mathrm{e}}(r)$. For example, the number of atoms in the interchain region within $4.8 \AA$ is 7.4. Herein, there exist the intrachain peaks of $g^{ \pm} g^{ \pm}$and $t g^{ \pm}$conformations, as predicted from Table I. If the rotational isomeric state model is adopted, the number of atoms of $g^{ \pm} g^{ \pm}$and $t g^{ \pm}$conformations are obtained as 0.2 and 1.2 from Table II. By subtracting $(0.2+1.2)$ from the number of atoms in the interchain region within $4.8 \AA(=7.4)$, the number of interchain atoms within $4.8 \AA$ is estimated to be 6.0. We are now applying a similar analysis to PE amorphotized by $\gamma$-ray irradiation. The interchain packing in the amorphous solid state of PE-like polymers will be discussed in greater detail in the near future.

Acknowledgment. The authers wish to thank M. Shiraishi of Japan E-P Rubber Co. for kindly supplying EPRC samples and information on characteristics of these samples. This work was supported in part by a Grant-in-Aid for Scientific Research from the Ministry of Education, Science and Culture of Japan.

\section{APPENDIX 1}

Conformational probabilities for an isolated EPRC chain in a $\theta$-solvent can be calculated using the rotational isomeric state model of Flory et al. ${ }^{12}$ There are three isomeric states in the skelton bonding, which are referred to as trans $(t)$, gauche plus $\left(g^{+}\right)$and gauche minus $\left(g^{-}\right)$. Their conformational probabilities are given by the statistical weight matrices $\mathbf{U}_{i}$, in which rows are associated with rotational states about the bond $i-1$ and columns with states about the bond $i$ : both rows and columns are indexed in the order $t, g^{+}, g^{-}$. According to Mark, ${ }^{13}$ various combinations of the statistical weight materices are used to represent all possible consecutive bond pairs in the EPRC chain (20 $\mathrm{mol} \%$ of propylene unit).

(I) For bond pairs $\mathrm{CH}_{2}-\mathrm{CH}_{2}-\mathrm{CH}_{2}$ : The statistical weight matrix has the form,

$$
\mathbf{U}_{e}=\left[\begin{array}{ccc}
1 & \sigma & \sigma \\
1 & \sigma & \omega \sigma \\
1 & \omega \sigma & \sigma
\end{array}\right]
$$

where the parameter $\sigma$ denotes the statistical weight applicable to a gauche state when the proceeding state is either trans or gauche of the same sign, this statistical weight being relative to the value of unity for the trans state. The factor $\omega$ takes account of the severe steric overlap of a pair of gauche states opposite in sign, (pentane effect).

(II) For a pair of bonds meeting at a $\mathrm{CHCH}_{3}$ group: The atomic configurations of the group are specified by the $d$ and $l$ conventions. ${ }^{12}$ The statistical weight matrix for the $d$ configuration is given by,

$$
\mathbf{U}_{d}=\left[\begin{array}{ccc}
\sigma & \sigma & \tau \sigma \\
\sigma & \sigma & \omega \tau \sigma \\
\sigma & \omega \sigma & \tau \sigma
\end{array}\right]
$$

where $\tau$ is the statistical weight of conformation in which $\mathrm{CH}$ or $\mathrm{CH}_{2}$ is syn to both $\mathrm{CH}_{2}$ and $\mathrm{CH}_{3}$. The matrix $\mathbf{U}_{l}$ associated with the $l$ configuration is obtained by the simple interchange of both the second and third rows and the second and third columns of $\mathbf{U}_{d}$.

(III) For pairs of bonds separating two $\mathrm{CHCH}_{3}$ groups: The statistical weight matrices for the dyads $d d$ and $d l$ are given by,

$$
\begin{aligned}
& \mathbf{U}_{d d}=\left[\begin{array}{ccc}
\omega \sigma & \omega \tau \sigma & \sigma \\
\sigma & \omega \tau \sigma & \omega \sigma \\
\omega \sigma & \omega^{2} \sigma \tau & \omega \sigma
\end{array}\right] \\
& \mathbf{U}_{d l}=\left[\begin{array}{ccc}
\sigma & \omega \sigma & \omega \tau \sigma \\
\omega \sigma & \sigma & \omega \tau \sigma \\
\omega \sigma & \omega \sigma & \omega^{2} \sigma \tau
\end{array}\right]
\end{aligned}
$$

The matrices $\mathbf{U}_{l l}$ and $\mathbf{U}_{l d}$ are obtained respectively from $\mathbf{U}_{d d}$ and $\mathbf{U}_{d l}$ by the interchanges of rows and columns.

(IV) Bond pairs $\mathrm{CHCH}_{3}-\mathrm{CH}_{2}-\mathrm{CH}_{2}$ and $\mathrm{CH}_{2}-$ $\mathrm{CH}_{2}-\mathrm{CHCH}_{3}$ : The former are characterized by $\mathbf{U}_{d e}$ and $\mathbf{U}_{l e}$, and the latter by $\mathbf{U}_{e d}$ and $\mathbf{U}_{e l}$, where, 


$$
\begin{aligned}
& \mathbf{U}_{d e}=\left[\begin{array}{ccc}
1 & \omega \sigma & \sigma \\
1 & \sigma & \omega \sigma \\
1 & \omega \sigma & \omega \sigma
\end{array}\right] \\
& \mathbf{U}_{e d}=\left[\begin{array}{ccc}
\sigma & \tau \sigma & \sigma \\
\sigma & \omega \tau \sigma & \omega \sigma \\
\omega \sigma & \omega \tau \sigma & \sigma
\end{array}\right]
\end{aligned}
$$

$\mathbf{U}_{l e}$ and $\mathbf{U}_{e l}$ are related to $\mathbf{U}_{d e}$ and $\mathbf{U}_{e d}$, respectively, by the interchange of rows and columns.

Calculations have been carried out by using the following values ${ }^{14,15}$ for $\sigma, \tau$, and $\omega$ at room temperature; $-R T \ln \sigma=500 \mathrm{cal} \mathrm{mol}^{-1}, \quad-R T \ln \tau=$ $450 \mathrm{cal} \mathrm{mol}^{-1}$, and $-R T \ln \omega=2000 \mathrm{cal} \mathrm{mol}^{-1}$. The configuration partition function $Z$ for the EPRC chain of $n$-bonds can thus be written as,

$$
Z=\mathbf{J} *\left[\prod_{i=2}^{n-1} \mathbf{U}_{i}\right] \mathbf{J}
$$

where $J^{*}=\left[\begin{array}{lll}1 & 0 & 0\end{array}\right]$ and $\mathbf{J}$ is the transpose of [ [lll 111$]$. The probability that the bond $i$ is in state $\eta\left(\eta=t, g^{+}\right.$ or $g^{-}$) is given by,

$$
P_{\eta ; i}=Z^{-1} \mathbf{J}^{*}\left[\prod_{h=2}^{i} \mathbf{U}_{h}\right] \mathbf{U}_{\eta ; i}^{\prime}\left[\prod_{j=i+1}^{n-1} \mathbf{U}_{j}\right] \mathbf{J}
$$

where $\mathbf{U}_{\eta ; i}^{\prime}$ is formed from the matrix $\mathbf{U}_{i}$ by retaining the column $\eta$ and replacing the elements in other columns by zero. The probability that the bond $i-1$ is in state $\xi$ and the bond $i$ in state $\eta$ is given by,

$$
P_{\xi \eta ; i}=Z^{-1} \mathbf{J} *\left[\prod_{h=2}^{i-1} \mathbf{U}_{h}\right] \mathbf{U}_{\xi \eta^{\prime} i}^{\prime}\left[\prod_{k=i+1}^{n-1} \mathbf{U}_{k}\right] \mathbf{J}
$$

where $\mathbf{U}_{\xi \eta, i}^{\prime}$ is formed from the matrix $\mathbf{U}_{i}$ by replacing all elements with zero except for element $\xi \eta$. The sequences of U's appearing in eq A7-A9 are specified by the chemical sequences of monomer units along a chain. By the Monte Carlo method, the random sequences of 500 monomers consisting of ethylene and propylene units with $4: 1(20 \mathrm{mo} \%$ of propylene unit) are generated in a chain.

$P_{\eta, i}$ and $P_{\xi \eta, i}$ for all bonds except 5 bonds on both ends of the chain are calculated. The averages of $P_{\eta, i}$ and $P_{\xi \eta, i}$ for all bonds are denoted by $\left(P_{t}, P_{g^{ \pm}}\right)$and $\left(P_{t t}, P_{t g^{ \pm}}, P_{g^{ \pm} g^{ \pm}}\right)$, respectively. Their values are shown in Table II.

\section{APPENDIX 2}

\section{Derivation of $h_{\mathrm{CH}}\left(r, r_{\mathrm{C}}\right)$}

We consider the radial distribution function of $\mathrm{H}$ atoms belonging to a $\mathrm{CH}_{x}$ unit ( $x=1$ to 3$)$, when $\mathrm{H}$ atoms are distributed in spherical symmetry about the $\mathrm{C}$ atom bonding to them. We assume that the number density of an $\mathrm{H}$ atom located at a distance $r^{\prime}$ from the $\mathrm{C}$ atom is expressed by a Gaussian function such as,

$$
v_{\mathrm{H}}\left(r^{\prime}\right)=1 / A \cdot \exp \left[-\left(r^{\prime}-r_{\mathrm{CH}}\right)^{2} / \Delta^{2}\right]
$$

and,

$$
\begin{aligned}
A= & 4 \pi \int_{0}^{\infty} \exp \left[-\left(r-r_{\mathrm{CH}}\right)^{2} / \Delta^{2}\right] r^{2} \mathrm{~d} r \\
= & \Delta \sqrt{\pi^{3}}\left(\Delta^{2}+2 r_{\mathrm{CH}}^{2}\right)+2 \pi \Delta^{2} r_{\mathrm{CH}} \exp \left(-r_{\mathrm{CH}}^{2} / \Delta^{2}\right) \\
& +2 \pi\left(\Delta^{2}+2 r_{\mathrm{CH}}^{2}\right) \int_{0}^{r_{\mathrm{CH}}} \exp \left(-z^{2} / \Delta^{2}\right) \mathrm{d} z
\end{aligned}
$$

where $r_{\mathrm{CH}}=1.09 \AA$ is a $\mathrm{C}-\mathrm{H}$ bond length and $\Delta=0.03 \AA$ is its amplitude of thermal vibration. When the $\mathrm{H}$ and $\mathrm{C}$ atoms are placed at $\vec{r}$ and $\vec{r}_{\mathrm{C}}$, $r^{\prime}$ is given by

$$
r^{\prime}=\sqrt{r^{2}+r_{\mathrm{C}}{ }^{2}-2 r \cdot r_{\mathrm{C}} \cdot \cos \theta}
$$

where $\theta$ is the angle between $\vec{r}$ and $\vec{r}_{\mathrm{C}}$. Following Hosemann et al., ${ }^{16}$ the conditional probability, that is, $\mathrm{RDF}$ of an $\mathrm{H}$ atom, is obtained by integrating eq A10 (when the bonding $\mathrm{C}$ atom is located at $r_{\mathrm{C}}$ ):

$$
\begin{aligned}
h_{\mathrm{CH}}\left(r, r_{\mathrm{C}}\right) \\
=(2 \pi / A) \int_{-1}^{1} \exp \left[-\left(\sqrt{r^{2}+r_{\mathrm{C}}^{2}-2 r \cdot r_{\mathrm{C}} \cdot \cos \theta}\right.\right. \\
\left.\left.\quad-r_{\mathrm{CH}}\right)^{2} / \Delta^{2}\right] \mathrm{d}(\cos \theta) \\
=\left(\Delta^{2} / r \cdot r_{\mathrm{C}} \cdot A\right)\left\{\exp \left[-\left(\left|r-r_{\mathrm{C}}\right|-r_{\mathrm{CH}}\right)^{2} / \Delta^{2}\right]\right. \\
\left.\quad-\exp \left[-\left(r+r_{\mathrm{C}}-r_{\mathrm{CH}}\right)^{2} / \Delta^{2}\right]\right\} \\
+2 \sqrt{2} r_{\mathrm{CH}} \int_{a_{1}}^{a_{2}} \exp \left[-\left(2 r \cdot r_{\mathrm{C}} \cdot z^{2} / \Delta^{2}\right)\right] \mathrm{d} z \quad(\mathrm{~A} 13)
\end{aligned}
$$

where,

$$
a_{1}=\left(\left|r-r_{\mathrm{C}}\right|-r_{\mathrm{CH}}\right) / \sqrt{2 r \cdot r_{\mathrm{C}}}
$$

and,

$$
a_{2}=\left(r+r_{\mathrm{C}}-r_{\mathrm{CH}}\right) / \sqrt{2 r \cdot r_{\mathrm{C}}}
$$

\section{REFERENCES}

1. I. Voigt-Martin and F. C. Mijlhoff, J. Appl. Phys., 46, 1165 (1975); I. Voigt-Martin and F. C. Mijlhoff, 
ibid., 47, 3942 (1976).

2. G. W. Longman, G. D. Wignall, and R. P. Sheldon, Polymer, 17, 485 (1976).

3. G. W. Longman, G. D. Wignall, and R. P. Sheldon, Polymer, 20, 1063 (1979).

4. S. Kan and T. Seto, Rep. Prog. Polym. Phys. Jpn., 19, 219 (1976).

5. O. Yoda, I. Kuriyama, and A. Odajima, Appl. Phys. Lett., 32, 18 (1978); O. Yoda, I. Kuriyama, and A. Odajima, J. Appl. Phys., 49, 5468 (1978).

6. M. Gupta and G. S. Yeh, J. Macromol. Si., Phys., B16(2), 225 (1978).

7. M. Numakawa and A. Odajima, Rep. Prog. Polym. Phys. Jpn., 22, 141 (1979).

8. P. G. Maket, C. Cabos, A. Escande, and P. Delord, J. Appl. Cryst., 6, 139 (1973).

9. C. N. J. Wagner, "Liquid Metals, Chemistry and Physics," S. Z. Beer, Ed., Marcel Dekker, New York,
1973, p 280.

10. C. H. Macgillavry and G. D. Rieck, Ed., "International Tables for X-ray Crystallography," Vol. III, Kynoch Press, Birmingham, England, 1962, p 202 and p 250.

11. B. E. Warren, "X-ray Diffraction," AddisonWelsley, Reading, Massachusetts, 1969, p 129.

12. P. J. Flory, "Statistical Mechanics of Chain Molecules,"'Interscience, New York, 1969, Chapters 3 and 6.

13. J. E. Mark, J. Chem. Phys., 57, 2541 (1972).

14. A. Abe, R. L. Jernigan, and P. J. Flory, J. Am. Chem. Soc., 88, 631 (1966).

15. U. W. Suter and P. J. Flory, Macromolecules, 8, 765 (1975).

16. R. Hosemann and S. N. Bagchi, "Direct Analysis of Diffaction by Matter," North Holland, Amsterdam, 1962, p. 69; S. N. Bagchi, Adv. Phys., 19, 119 (1970). 